\title{
Coherencia interna de diseños curriculares de educación inicial en Latinoamérica en relación con su enfoque de aprendizaje
}

\section{Lulin tinkuy akapap yaćhana yaćhanan maśhtaykuna latinu amirikaćhu yaćhanan achikyaninwan}

\section{Okantingatagetanë kengagantsi irasijanekijani kara timagantsigitekë ratinoamerika kara okanta iragotaneegi janekijanipage obamentotsipongokë}

\author{
Recibido: 04 enero 2020 Corregido: 15 marzo 2020 Aprobado: 22 abril 2020 \\ Ivvanova De Jesús Dávila Padrón \\ Nacionalidad: Venezuela / Universidad Católica Andrés Bello, Venezuela \\ Correo: ijdavila.11@est.ucab.edu.ve /Orcid: http://orcid.org/oooo-0003-0468-9311 \\ Marcos Antonio Requena Arellano \\ Nacionalidad: Venezolana / Universidad Siglo 21, Argentina. \\ Correo: marcos.requena@ues21.edu.ar /ORCID: http://orcid.org/oooo-0oo3-2679-5270
}

\section{Resumen}

Se presenta un análisis de la coherencia interna de los diseños curriculares de Educación Inicial de los nueve países suramericanos hispanohablantes, en relación con su enfoque de aprendizaje. Previamente al análisis, se señala el concepto de currículo y de consistencia interna en que el mismo se basa; asimismo, se expone la clasificación asumida de los enfoques de aprendizaje, la que distingue tres fundamentales: conductismo, cognitivismo y constructivismo. Se aplicó un método mixto (cualicuantitativo) con diseño secuencial. Se emplean dos instrumentos y dos escalas. Hallazgos: a) cinco diseños curriculares explicitan su enfoque de aprendizaje; b) de estos cinco documentos, tres son altamente coherentes con dicho enfoque y dos son medianamente coherentes; c) de los cuatro diseños curriculares que no explicitan algún enfoque de aprendizaje, tres contienen evidencias de tres enfoques, y el cuarto documento contiene evidencias de dos enfoques; d) dos de estos cuatro documentos presentan alta coherencia, y los otros dos presentan baja coherencia. Se concluye que la coherencia curricular, en relación con el enfoque de aprendizaje referente, se presenta de manera diferenciada en los distintos diseños curriculares del conjunto estudiado.

\section{Palabras clave:}

Currículo preescolar, diseño curricular, evaluación curricular; investigación curricular; teorías de aprendizaje.

\section{Lisichiku limaykuna:}

Manalayaćhapakup yaćhanan, yaćhana maśhtay, yaćhaykuna tapukuy, yaćhaykuna ashipana, yaćhanap yaćhayninkuna.

\section{Nibarintsipage katingatsaro:} sangenarentsi kantëgotiro ikokagani janekijani, monkaratagantsi, aike ora sangenarentsipage niaokoaeiri janekijanipage kara igoigagetiri. 


\title{
Internal Coherence of Curricular Designs of Kindergarden Education in Latin America whit Relation to its Learning Approach
}

\begin{abstract}
An analysis of the internal coherence of the Initial Education curricular designs of the nine Spanish-speaking South American countries is presented, in relation to their learning approach. Before the analysis, the concept of curriculum and internal consistency on which it is based is pointed out; Likewise, the assumed classification of learning approaches is exposed, which distinguishes three fundamental ones: behaviorism, cognitivism and constructivism. A mixed method (qualitative and quantitative) with sequential design was applied. Two instruments and two scales are used. Findings: a) Five curricular designs explain your learning approach; b) of these five documents, three are highly consistent with that approach and two are fairly consistent; c) of the four curricular designs that do not explain any learning approach, three contain evidence of three approaches, and the fourth document contains evidence of two approaches; d) two of these four documents have high coherence, and the other two have low coherence. It is concluded that the curricular coherence, in relation to the referent learning approach, is presented in a different way in the different curricular designs of the studied group.
\end{abstract}

\author{
Keywords \\ Curriculum \\ Evaluation; Preschool \\ Curriculum; \\ Curriculum Research, \\ Learning Theories; \\ Curriculum Design
}

\section{Coerência interna dos desenhos curriculares da educação inicial na América Latina em relação à sua abordagem à aprendizagem}

\section{Resumo}

Apresenta-se uma análise da coerência interna dos desenhos curriculares da Educação Inicial dos nove países sul-americanos de língua espanhola em relação à sua abordagem de aprendizagem. Prévio à análise, destaca-se o conceito de currículo e consistência interna no qual se baseia; Da mesma forma, expõe-se a classificação atribuída dos enfoques da aprendizagem, que distingue três fundamentais: comportamentalismo, cognitivismo e construtivismo. Foi aplicado um método misto (qualiquantitativo) com desenho sequencial. São utilizados dois instrumentos e duas escalas. Resultados: a) Cinco desenhos curriculares explicam sua abordagem de aprendizagem; b) desses cinco documentos, três são altamente consistentes com essa abordagem e dois são de mediana consistência; c) dos quatro desenhos curriculares que não explicam claramente algum enfoque da aprendizagem, três contêm evidências de três enfoques e o quarto documento contém evidências de dois enfoques; d) dois desses quatro documentos apresentam alta coerência, e os outros dois têm baixa coerência. Conclui-se que a coerência curricular, em relação com o enfoque da aprendizagem referencial, é apresentada de forma diferente nos diferentes desenhos curriculares do conjunto estudado.

\author{
Palavras-chave: \\ Currículo pré- \\ escolar, desenho \\ curricular, avaliação \\ curricular, pesquisa \\ curricular, teorias da \\ aprendizagem.
}

\begin{abstract}
Datos de los autores
Ivvanova De Jesús Dávila Padrón. Magister en Educación - mención Procesos de Aprendizajes y Licenciada en Educación, por la Universidad Católica Andrés Bello, Venezuela. Línea de investigación centrada en diseño curricular de educación inicial y procesos de aprendizajes y enseñanza en dicho nivel. Directora de Jardín Maternal e Inicial "I love My Kinder", Montevideo - Uruguay.

Marcos Antonio Requena Arellano. Doctor en Educación y Magister en Psicología Cognitiva por la Universidad Católica Andrés Bello, Venezuela. Licenciado en Filosofía por la Universidad Central de Venezuela. Atención de investigación centrada en formación docente, educación basada en competencias, educación digital, procesos de aprendizaje y autorregulación académica. Coordinador de investigación del área de Ciencias Humanas y Sociales de la Universidad Siglo 21, Argentina. Profesor a distancia del Doctorado en Educación y Maestría en Procesos de Aprendizaje de la Universidad Católica Andrés Bello, Venezuela.
\end{abstract}




\section{Introducción}

La Convención sobre los Derechos del Niño, del Fondo de las Naciones Unidas para la Infancia (Unicef, 1989) establece un conjunto universalmente aceptado de normas y obligaciones que dan a los niños un papel protagónico en la construcción de una sociedad justa, respetuosa y pacífica. Posteriormente, en 1990, se lleva a cabo la Cumbre Mundial a favor de la Infancia, teniendo como objetivo hacer un Ilamado urgente a nivel mundial para brindar un futuro mejor a los niños. Entre sus compromisos contempla la ejecución de ofrecer oportunidades de educación para todos los niños (Unicef, 1990). Por otra parte, la Declaración Mundial Sobre Educación para Todos (Conferencia Mundial de Educación para todos [WCEFA], 1990) enfatiza la importancia de la educación inicial de la infancia.

En el marco de esta intencionalidad de ofrecer un servicio educativo de calidad para la población infantil, se ha resaltado la importancia de contar con un diseño instruccional que sirva de orientador. Blanco (2005) sostiene que para el desarrollo integral del infante en contexto educativo "es preciso desarrollar modelos e instrumentos acordes a la naturaleza e idiosincrasia de esta etapa educativa" (p.25). Asimismo, Casanova (2012) expone que "cualquier mejora de la calidad educativa pasa por su traducción al diseño curricular que es lo que, efectivamente, llega a centros y aulas, es decir, a los estudiantes de cada época histórica" (p.8).

Una variedad de autores señalan las implicaciones del diseño curricular en la calidad educativa, comprendiéndose el currículo como una herramienta guía y de control del proceso educativo (Badilla, 2009; Blanco, 2005; Castañeda, Castro \& Mena, 2012; Freire, Páez, Núñez, Narváez \& Infante, 2018; Inciarte \& Canquiz, 2001; Martínez Carpio, 2013; Mata, 2015; Salas Perea, 2016; Sánchez, 2015; Terigi, 2002). Tal papel del currículo le atribuye marcada importancia a la evaluación curricular, y entre los autores existe un gran consenso en que los criterios de dicha evaluación son, fundamentalmente cuatro: relevancia, pertinencia, significación y consistencia. La relevancia se refiere al para qué y qué de la educación -fines y contenidos-; la pertinencia hace referencia a la diversidad cultural desde la adecuación y adaptación de materias al contexto de vida, y la significación está referida a los intereses de los alumnos. Inciarte y Canquiz (2001) definen la consistencia interna de un currículo como la "relación interna de solidez, complementariedad, ausencia, repetición, semejanza y analogía de los elementos que conforman una estructura curricular" (p.7). Varios son los elementos o criterios que definen esta consistencia; uno de ellos es el de la coherencia: "la relación de unión, correspondencia o articulación entre los diferentes elementos que conforman el currículo" (Inciarte y Canquiz, 2001, p. 8).

En lo referente a la Educación Inicial, se han hecho críticas a la orientación que con frecuencia se observa en los hechos: "muchos de los programas de este nivel educativo se han caracterizado más por 'cuidar' y 'guardar' a los niños que por su intencionalidad educativa. (Arancibia, Blanco \& Avilés, 2004, p.7). Las críticas se centran fundamentalmente en el criterio de que la Educación Inicial es un nivel educativo, no un servicio para cuidar niños (Arancibia, Blanco \& Avilés, 2004; Falabella, Cortázar, Godoy, González \& Romo 2018; Luzón \& Montes, 2018). Dada la ya señalada implicación del diseño curricular en la calidad educativa, resulta relevante realizar investigaciones analíticas de los documentos curriculares de la Educación Inicial; estos son los documentos oficiales por los cuales se rigen las distintas instituciones que brindan educación a la primera infancia.

En relación con la consistencia interna que se espera de todo diseño curricular y del enfoque educativo que este concreta, Jiménez (2008) plantea que es importante analizar la con- 
cepción sobre lo qué es la educación y sus funciones, las distintas relaciones entre los sujetos señalados en el currículo (docentes, alumnos...), las características de sus roles, el uso que se hace de los recursos de aprendizaje, los contenidos contemplados y las estrategias y uso de la evaluación.

Dado que el núcleo de la educación es el aprendizaje que se espera sea logrado por el alumno, no es de extrañar que los distintos componentes del currículo manifiesten de algún modo una concepción de aprendizaje; como sostiene Schunk (2012), “la teoría proporciona un marco de referencia para la toma de decisiones educativas" (p.20). Cada uno de los componentes del diseño curricular se orienta de acuerdo con la concepción de aprendizaje que explícita o implícitamente lo anima. Ertmer \& Newby (2013) y Jiménez, (2008) sostienen que todo diseño instruccional propone la implementación de un enfoque de aprendizaje y que tal implementación se verifica en componentes como: fines del hecho educativo, papel del docente, papel del alumno, secuenciación del aprendizaje, estrategias de enseñanza, estrategias de aprendizaje, estrategias de evaluación.

Con base en lo anterior, los autores del presente artículo, interesados de manera particular por la educación infantil en América Latina, se preguntaron sobre la existencia de reportes de investigaciones orientadas al análisis de la consistencia interna de los currículos de Educación Inicial en dicha región. El centro de interés de los investigadores estaba en dos aspectos relevantes de la evaluación curricular, señalados anteriormente: a) la consistencia interna de los currículos de Educación Inicial y b) el sustento teórico de dichos diseños curriculares, en lo que respecta al enfoque de aprendizaje. Para responderse la pregunta señalada, se hizo una búsqueda en los directorios Dialnet, Latindex, Redalyc y Scielo, de revistas científicas de acceso abierto, publicadas entre 2009 y 2018 por editoriales hispanoamericanas pertenecientes a instituciones educativas. No se encontró ningún artículo que abordara dicho tema con tal alcance geográfico o, al menos, con alcance de dos o más países de la mencionada región.

Con base en la indicada búsqueda fallida, los autores del presente artículo consideraron pertinente y de valor realizar una investigación a fin de examinar la coherencia interna de los documentos curriculares de educación inicial de los países suramericanos de habla hispana, en lo que respecta a sus fundamentos teóricos en materia de aprendizaje. De manera precisa, se buscó determinar, para cada documento curricular, la coherencia entre la fundamentación teórica respecto del aprendizaje y los planteamientos orientadores (fines y objetivos) y prácticos: rol docente, rol del estudiante, estrategias de enseñanza, estrategias de aprendizaje y estrategias de evaluación.

\section{Método}

La investigación se enmarcó en el enfoque mixto: se emplearon técnicas de recolección y análisis de datos, primeramente, cualitativos y posteriormente cuantitativos (Hernández, Fernández \& Baptista, 2010). Por su nivel de profundidad, la indagación fue de tipo evaluativo: se buscó determinar el nivel de coherencia interna de los diseños curriculares de acuerdo con el enfoque de aprendizaje contenido.

La indagación se llevó a cabo mediante un diseño mixto secuencial cuali-cuantitativo (Edmonds \& Kennedy (2013): una primera fase con método cualitativo, seguida de una con método cuantitativo. En la primera etapa se aplicó una técnica de análisis cualitativo de contenido, siguiendo un procedimiento adaptado de Cáceres (2003): 


\section{Fase 1. Cualitativa}

PASOS PRE-ANALÍTICOS. Previamente a la aplicación de la Fase 1, y para su preparación, se aplicaron los siguientes seis pasos:

1. Selección del objeto de análisis: el documento curricular, al nivel de las aseveraciones, descripciones o prescripciones explícitas.

2. Definición de la unidad de análisis: frases con unidad gramatical -descripciones, aseveraciones o prescripciones, encontradas en los diseños curriculares.

3. Pre-análisis: colección de documentos: los diseños curriculares de Educación Inicial de los nueve países de habla hispana de Suramérica: Argentina, Bolivia, Colombia, Chile, Ecuador, Paraguay, Perú, Uruguay y Venezuela. (véase Tabla 1).

Tabla 1

Documentos curriculares objeto de análisis

\begin{tabular}{|c|c|c|}
\hline País & Documento & Referencia \\
\hline Argentina & $\begin{array}{l}\text { Diseño Curricular para la Educación Inicial. } \\
\text { Marco General. }\end{array}$ & $\begin{array}{l}\text { Secretaría de Educación de la } \\
\text { Ciudad Autónoma de Buenos } \\
\text { Aires. (2000). }\end{array}$ \\
\hline Bolivia & $\begin{array}{l}\text { Diseño Curricular para el Nivel de Educación } \\
\text { Inicial. }\end{array}$ & $\begin{array}{l}\text { Ministerio de Educación de } \\
\text { Bolivia.(2012) }\end{array}$ \\
\hline Colombia & $\begin{array}{l}\text { Bases Curriculares para la Educación Inicial y } \\
\text { Preescolar. }\end{array}$ & $\begin{array}{l}\text { Ministerio de Educación de } \\
\text { Colombia (2017). }\end{array}$ \\
\hline Chile & Bases Curriculares de la Educación Parvularia. & $\begin{array}{l}\text { Ministerio de Educación de } \\
\text { Chile. (2018) }\end{array}$ \\
\hline Ecuador & Currículo de Educación Inicial. & $\begin{array}{l}\text { Ministerio de Educación de } \\
\text { Ecuador. (2014). }\end{array}$ \\
\hline Paraguay & Marco Curricular de la Educación Inicial & $\begin{array}{l}\text { Ministerio de Educación y } \\
\text { Cultura de Paraguay. (2005). }\end{array}$ \\
\hline Perú & Currículo Nacional de la Educación Básica. & $\begin{array}{l}\text { Ministerio de Educación de Perú. } \\
(2016) \text {. }\end{array}$ \\
\hline Uruguay & $\begin{array}{l}\text { Marco Curricular para la atención y } \\
\text { educación de niñas y niños uruguayos desde } \\
\text { el nacimiento a los seis años. }\end{array}$ & $\begin{array}{l}\text { Ministerio de Educación } \\
\text { Cultura de Uruguay. (2014) }\end{array}$ \\
\hline Venezuela & $\begin{array}{l}\text { Guía pedagógica - didáctica Educación inicial } \\
\text { Etapa Preescolar }\end{array}$ & $\begin{array}{l}\text { Ministerio del Poder Popular } \\
\text { para la Educación de Venezuela. } \\
\text { (2013) }\end{array}$ \\
\hline
\end{tabular}

Como se observa en la Tabla 1, el conjunto de diseños curriculares objeto de análisis datan de un período que va de 2012 a 2016, teniendo, así, el más antiguo (de Bolivia) siete años de vigencia, y el más reciente (de Chile), un año.

4. Formulación de la guía de trabajo: conjunto de categorías previas, organizadas por dimensiones curriculares, con base en las manifestaciones curriculares de los enfoques de aprendizaje conductista, cognitivista y constructivista; ello, según exposiciones de Ertmer \& Newby (2013). Las categorías fueron: a. Enfoque de Aprendizaje, b. Fines del proceso educativo, c. Estrategias de Enseñanza, d. Estrategias de Aprendizaje, e. Rol docente, f. Estrategias de Evaluación. La técnica para esta etapa fue el análisis cualitativo de contenido. 
5. Establecimiento de indicadores de dichas categorías, para los enfoques:

a) Conductista, según exposiciones de Cadena \& Saucedo (2016), Ertmer \& Newby (2013), Hernández (2002), Manterola (2012), Poggioli (1997) y Valdez (2012).

b) Cognitivista, según Ertmer \& Newby (2013), Koumakhov \& Daoud (2017), Gutiérrez Ruiz (2015), Marugán, Martín, Catalina \& Román (2013).

a) Constructivista, según Carretero, (2009), Cormack (2004), Ertmer \& Newby (2013), Guerrero \& Flores (2009), Manterola (2012) y Serrano \& Pons (2011).

Las Tablas 2, 3 y 4 presentan los productos de los pasos 3 y 4 de la Fase 1 . Se trata de las dimensiones e indicadores de presencia en un diseño curricular de, respectivamente, los enfoques de aprendizaje conductista, cognitivista y constructivista. Con base en tales dimensiones e indicadores se construyeron los instrumentos para la realización del análisis cualitativo de los diseños curriculares.

Tabla 2

Criterios e indicadores de la presencia del enfoque conductista del aprendizaje en un documento curricular

\begin{tabular}{ll}
\hline \multicolumn{1}{c}{ Criterio } & \multicolumn{1}{c}{ Indicadores } \\
\hline Fin u objetivo de la educación & Planteamientos orientadores \\
\hline Roles del docente & Planteamientos prácticos \\
\hline Estrategias de enseñanza & Onálisis conductual de tareas \\
& Administración de estímulos \\
& Administración de reforzadores \\
\hline Estrategias de aprendizaje & Secuenciación de contenido con complejidad progresiva \\
& Uso de pistas o indicios \\
& Modelaje \\
& Retroalimentación reforzadora \\
\hline Estrategias de evaluación & Transferencia mediante generalización \\
& Uso de señales instruccionales \\
& Práctica de fortalecimiento de asociaciones \\
\hline & Métodos cuantitativos \\
& Establecimiento de línea base \\
& Técnicas de identificación y análisis conductual \\
& Evaluación continua de resultados - esquema de \\
aproximación sucesiva
\end{tabular}

Como se expresa en la Tabla 2, existe un mayor número (5) de evidencias de indicadores para el criterio Estrategias de Evaluación en comparación al resto. Con igual proporción de indicadores (4), se encuentran los criterios del Rol del docente y Estrategias de Enseñanza. Para la categoría Estrategias de aprendizaje se hallaron (3) indicadores. Se hace notable, así, que este enfoque le asigna un mayor peso aspectos asociados al docente (enseñanza y evaluación) que a aspectos del estudiante (estrategias de aprendizaje). 
Tabla 3

Criterios e indicadores de la presencia del enfoque cognitivista del aprendizaje en un documento curricular.

\begin{tabular}{ll}
\hline \multicolumn{1}{c}{ Criterio } & \multicolumn{1}{c}{ Indicadores } \\
\hline Fin u objetivo de la educación & Planteamientos orientadores \\
\hline Roles del docente & Planteamientos prácticos \\
\hline & Análisis cognitivo de tareas \\
& Análisis de la disposición del alumno para el aprendizaje \\
& significativo \\
& Animación al alumno \\
& Organización de la información para que sea potencialmente \\
& significativa \\
& Diseño de la formación de acuerdo con el conocimiento \\
& previo del alumno \\
& Transmisor de conocimiento \\
\hline Estrategias de enseñanza & Simplificación y agrupación de la información \\
& Explicaciones instruccionales \\
& Ejemplos demostrativos, analogías \\
& Retroalimentación informativa \\
\hline Estrategias de apoyo y motivacionales \\
Estrategias cognitivas: esquematización, resumen, síntesis, \\
organizadores... \\
Estrategias de elaboración de información \\
Estrategias de organización de información \\
Metacognición: planificación, monitoreo, revisión \\
Integración métodos cuantitativos y cualitativos \\
Identificación del conocimiento previo del allumno de evaluación \\
Técnicas que permiten identificar la estructura conceptual y \\
los procesos cognitivos del alumno \\
Evaluación de procesos y resultados \\
\hline
\end{tabular}

En la Tabla 3 se observa igual proporción de indicadores (4) para las Estrategias de Enseñanza como para las Estrategias de Evaluación. Para las Estrategias de Aprendizaje se presenta (5) indicadores. Ello señala, en alguna medida, un cambio en relación al conductismo, en la atribución de importancia a acciones del docente y del alumno en el proceso educativo: aunque continúa dándose mayor importancia a las acciones del docente -sobre todo porque la evaluación sigue siendo responsabilidad fundamental de este-, se asignan al estudiante un papel más estratégico en el aprendizaje. 
Tabla 4

Criterios e indicadores de la presencia del enfoque constructivista del aprendizaje en un documento curricular.

\begin{tabular}{ll}
\hline \multicolumn{1}{c}{ Criterio } & \multicolumn{1}{c}{ Indicadores } \\
\hline Fin u objetivo de la educación & Planteamientos orientadores \\
\hline & utilidad personal. \\
\hline Roles del docente & Planteamientos prácticos \\
\hline & Identificación y análisis de los contextos complejos y \\
problemáticos del alumno & Guía en la resolución de problemas auténticos \\
& Mediación de aprendizajes orientados a -y logrados por- la \\
& resolución de problemas \\
\hline Estrategias de enseñanza & Planteamiento de situaciones problemáticas auténticas \\
& Activación del conocimiento previo: lluvia de ideas, \\
& formulación de preguntas \\
& Orientaciones sobre el proceso de solución de problemas \\
& Negoaciación social: debate, foro... \\
\hline Estrategias de aprendizaje & Formulación de hipótesis \\
& Interrogación activa sobre contenidos \\
& Aprendizaje colaborativo (incluye el juego) \\
& Reflexión sobre la experiencia \\
\hline Estrategias de evaluación & Métodos cualitativos \\
& Contextualización en situaciones problemáticas \\
& Basamento en el trabajo colaborativo \\
& Asignación de un rol relevante a la autoevaluación \\
\hline
\end{tabular}

La Tabla 4 muestra igualdad en la distribución de indicadores (4) para los criterios Estrategias de Enseñanza, Estrategias de Aprendizaje y Estrategias de Evaluación. Además de esta igualdad en estos tres aspectos, se observa, en relación con los enfoques cognitivista y conductista (principalmente con este último) un notable avance en la atribución de responsabilidades al alumno: en las estrategias de evaluación, se contemplan dos indicadores asociados con la actuación de este; son: el basamento en el trabajo colaborativo y la asignación de un rol relevante a la autoevaluación.

6. Elaboración de instrumentos de registros. Los instrumentos elaborados fueron:

A. Instrumento de análisis de coherencia enfoque - elementos curriculares: destinado a determinar en cada diseño curricular que tuviera explícito el enfoque de aprendizaje que le sustenta, el nivel de coherencia entre dicho enfoque, por una parte, y los planteamientos orientadores (fines y objetivos) y prácticos, por otra: estrategias de enseñanza, estrategias de aprendizaje, rol docente y estrategias de evaluación. Dado que el instrumento se aplicaría a los diseños curriculares que explicitan su enfoque de aprendizaje, se construyó tres versiones del mismo: uno por cada enfoque de aprendizaje.

B. Instrumento de análisis de coherencia intrateoría: destinado a determinar en cada diseño curricular que no tuviera explícito el enfoque de aprendizaje que le sustenta, el nivel de correspondencia de los planteamientos orientadores y prácticos del currículo 
con las proposiciones del enfoque de aprendizaje implícito predominante. Dado que el instrumento se aplicaría a los diseños curriculares que no explicitan su enfoque de aprendizaje, el mismo se construyó con la suma de indicadores de los tres enfoques de la taxonomía asumida en la investigación.

6. Validación de los instrumentos. Se aplicó una técnica de validación de contenido: juicio de expertos, solicitándose para cada indicador opinión sobre dos aspectos: coherencia y relevancia. Se sometieron a la opinión de diez (10) expertos, siguiendo la recomendación de Escobar y Cuervo (2008). Para la selección de los jueces, se siguieron los criterios propuestos por Escobar y Cuervo (2008): experiencia sobre el tema, reputación, disponibilidad, motivación e imparcialidad y adaptabilidad. Luego se determinó el nivel de concordancia de los juicios emitidos, con $w$ de Kendall .78 $\alpha=.05$. Se revisaron los indicadores y la redacción de las evidencias en las que coincidieron críticamente observaciones de expertos.

PASO ANALÍTICO. Paso de la Fase 1, propiamente dicho. Fue el siguiente:

7. Se empleó el instrumento correspondiente (para documento que explicita el enfoque de aprendizaje que le sustenta y para documento que no hace tal explicitación) para registrar, de cada documento, los fragmentos constitutivos de evidencias de los indicadores de los enfoques de aprendizaje. Se revisó la totalidad de los nueve diseños curriculares de Educación Inicial de la población.

\section{Fase cuantitativa}

Luego de la aplicación del instrumento respectivo a cada documento que explicita su enfoque de aprendizaje, se aplica la escala expuesta en la Tabla 5, con fines de determinar el nivel de coherencia interna del documento.

Tabla 5

Escala de nivel de coherencia interna de diseños curriculares con enfoque de aprendizaje explícito

\begin{tabular}{|c|c|}
\hline Nivel de coherencia & Caso en que se asigna \\
\hline Nula o casi nula coherencia & Se encuentran evidencias en $0 \%$ a $20 \%$ de indicadores \\
\hline Baja coherencia & $\begin{array}{l}\text { Se encuentran evidencias en } 21 \% \text { a } 40 \% \text { de } \\
\text { indicadores }\end{array}$ \\
\hline Mediana coherencia & $\begin{array}{l}\text { Se encuentran evidencias en } 41 \% \text { a } 60 \% \text { de } \\
\text { indicadores }\end{array}$ \\
\hline Alta coherencia & $\begin{array}{l}\text { Se encuentran evidencias en } 61 \% \text { a } 80 \% \text { de } \\
\text { indicadores }\end{array}$ \\
\hline Completa o casi completa coherencia & $\begin{array}{l}\text { Se encuentran evidencias en } 81 \% \text { a } 100 \% \text { de } \\
\text { indicadores }\end{array}$ \\
\hline
\end{tabular}

La escala presentada en la Tabla 5 responde al hecho de que los documentos curriculares a la que se destina su aplicación presentan de forma explícita el enfoque de aprendizaje en el que se sustentan. Es por ello que la diferencia entre los porcentajes de indicadores correspondientes a cada uno de los niveles de coherencia y el nivel contiguo, es siempre el mismo: $20 \%$ entre los mínimos porcentajes y $20 \%$ entre los máximos porcentajes de indicadores. 
Por otra parte, se aplica el instrumento correspondiente a los diseños cuyo enfoque de aprendizaje no se encuentra explícito, y posteriormente se emplea la escala expuesta en la Tabla 6, con fines de determinar el nivel de coherencia interna de tales documentos.

\section{Tabla 6 \\ Escala de nivel de coherencia interna de diseños curriculares con enfoque de aprendizaje no explícito.}

\begin{tabular}{ll}
\hline \multicolumn{1}{c}{ Nivel de coherencia } & \multicolumn{1}{c}{ Caso en que se asigna } \\
\hline & $\begin{array}{l}\text { En forma aproximadamente equilibrada, se identifican evidencias de } \\
\text { indicadores pertenecientes a dos enfoques no afines entre sí: con- } \\
\text { ductismo y cognitivismo, o conductismo y constructivismo; o entre } \\
\text { los tres enfoques (conductismo, cognitivismo y constructivismo). } \\
\text { En caso de existencia de evidencias de dos enfoques, las medidas } \\
\text { aproximadas de los indicadores de cada enfoque es 50\%; en caso de } \\
\text { existencia de evidencias de tres enfoques, las medidas aproximadas } \\
\text { de los indicadores de cada enfoque es 33\%. }\end{array}$ \\
& $\begin{array}{l}41 \% \text { a } 60 \% \text { de los indicadores con evidencias pertenecen a un en- } \\
\text { foque y el resto (40\% a 59\%) a los otros dos enfoques o solo a un } \\
\text { enfoque no afín. }\end{array}$ \\
Baja coherencia & $\begin{array}{l}41 \% \text { a } 60 \% \text { de los indicadores con evidencias pertenecen a un enfo- } \\
\text { que y el resto (40\% a 59\%) a un enfoque afín. } \\
\text { Mediana coherencia }\end{array}$ \\
Alta coherencia & foque. $80 \%$ de los indicadores con evidencias pertenecen a un en- \\
Completa o casi completa & $80 \%$ a $100 \%$ de los indicadores con evidencias pertenecen a un en- \\
foherencia &
\end{tabular}

Como muestra la Tabla 6, para los documentos curriculares con el enfoque de aprendizaje no explícito, los casos en que se asigna cada nivel de coherencia de la escala empleada deben considerar la posibilidad de que el currículo presente indicadores asociados con uno, dos o tres enfoques. Así, entonces, el nivel de coherencia a atribuir depende de las proporciones de los posiblemente encontrados indicadores de cada enfoque. Nótese que, consistentemente con este razonamiento, una nula coherencia se encontraría en un documento curricular en el que se encuentran indicadores de dos enfoques, con una proporción aproximada de $50 \%$ de cada grupo de indicadores; o en un documento en que se presentan indicadores de tres enfoques, con una proporción aproximada de $33 \%$ de cada grupo. Así va la escala hasta el máximo valor (completa o casi completa coherencia), el que se asigna si el 80\% o más de las evidencias corresponden a un enfoque en particular.

\section{Resultados y discusión}

Se encontró que cinco (5) de los diseños curriculares manifiestan explícitamente sustentarse en un enfoque de aprendizaje: el constructivismo. Los mismos son los documentos de Argentina, Bolivia, Paraguay, Perú y Uruguay. La Tabla 7 presenta evidencias de la presentación explícita de los enfoques de aprendizaje en los referidos cinco (5) diseños curriculares. Como puede verse, en los cinco diseños curriculares registrados se hace explícito que los mismos se sustentan en el enfoque de aprendizaje constructivista. En uno de ellos, el de Perú, se precisa que tal enfoque es el constructivismo social. 
Tabla 7

Síntesis de Documentos Curriculares con Enfoque de Aprendizaje explícito

\begin{tabular}{|c|c|c|}
\hline $\begin{array}{l}\text { Enfoque de } \\
\text { Aprendizaje } \\
\text { Explícito }\end{array}$ & País & Parte del texto que lo explicita \\
\hline \multirow{5}{*}{ Constructivismo } & Argentina & $\begin{array}{l}\text { Se privilegia, asimismo, una concepción constructivista del } \\
\text { conocimiento, la importancia de la interacción con los otros (p. 19) }\end{array}$ \\
\hline & Bolivia & $\begin{array}{l}\text { El abordaje de cada una [de las áreas] responde, en sus } \\
\text { planteamientos generales, al enfoque constructivista en los } \\
\text { procesos educativo (p.32) }\end{array}$ \\
\hline & Paraguay & $\begin{array}{l}\text { La metodología propuesta se enmarca dentro de la planteada por } \\
\text { la Reforma Educativa. Se fundamenta en posturas constructivistas } \\
\text { que posibilitan la construcción de saberes (p. } 35 \text { ) }\end{array}$ \\
\hline & Perú & $\begin{array}{l}\text { En ese sentido, se han definido orientaciones para aplicar } \\
\text { el enfoque pedagógico del Currículo Nacional de la } \\
\text { Educación Básica, las cuales se enmarcan en las corrientes } \\
\text { socioconstructivistas del aprendizaje (p. 171) }\end{array}$ \\
\hline & Uruguay & $\begin{array}{l}\text { Teniendo en cuenta la creciente complejidad de las áreas del } \\
\text { conocimiento y la especificidad disciplinar presente a lo largo del } \\
\text { proceso educativo, se enmarca en el enfoque constructivista de la } \\
\text { enseñanza (p. 14) }\end{array}$ \\
\hline
\end{tabular}

La Tabla 8 informa los niveles de coherencia de los cinco referidos diseños curriculares, cuyo enfoque de aprendizaje está explícito. Presenta, por documento: a) el número de indicadores en los que se encontraron evidencias; b) el número de indicadores en los que las evidencias son constructivistas; c) el nivel de coherencia del documento.

Tabla 8

Nivel de coherencia interna de los diseños curriculares con enfoque de aprendizaje explícito.

\begin{tabular}{|c|c|c|c|c|c|}
\hline $\begin{array}{c}\text { Documento } \\
\text { curricular/indicadores }\end{array}$ & $\mathrm{Ar}$ & Bo & $\mathrm{Pa}$ & $\mathrm{Pe}$ & Ur \\
\hline $\begin{array}{l}\text { Indicadores con } \\
\text { evidencias }\end{array}$ & 18 & 19 & 20 & 19 & 20 \\
\hline $\begin{array}{c}\text { Indicadores } \\
\text { con evidencias } \\
\text { constructivistas }\end{array}$ & $\begin{array}{c}11 \\
61 \%\end{array}$ & $\begin{array}{c}12 \\
63 \%\end{array}$ & $\begin{array}{c}11 \\
55 \%\end{array}$ & $\begin{array}{c}12 \\
63 \%\end{array}$ & $\begin{array}{c}9 \\
45 \%\end{array}$ \\
\hline Nivel de coherencia & Alta & Alta & Mediana & Alta & Mediana \\
\hline
\end{tabular}

Documentos: Ar. Argentina, Bo. Bolivia, Pa. Paraguay, Pe. Perú, Ur. Uruguay

Como muestra la Tabla 8, diseños curriculares de los cinco países presentan coherencia entre sus elementos prácticos y orientadores, por una parte, y el enfoque de aprendizaje en el que aseveran sustentarse: el constructivismo. No obstante, se puede observar diferencias en los niveles de coherencia: los documentos de Argentina (61\%), Bolivia (63\%) y Perú (63\%) arrojan un Alto nivel de coherencia, es decir, que entre el $61 \%$ y $80 \%$ de los indicadores presentan evidencias del enfoque constructivista, mientras que los documentos de Paraguay (55\%) y Uruguay (45\%) tienen una Mediana Coherencia entre las evidencias encontradas y el enfoque que explicitan. 
Se encontró que cuatro (4) de los nueve documentos analizados no presentan el enfoque de aprendizaje de manera explícita, siendo estos los de Chile, Colombia, Ecuador y Venezuela, pero, de forma explícita manifiestan planteamientos propios de más de un enfoque de aprendizaje.

La Tabla 9 muestra los resultados relativos a las evidencias de los indicadores correspondientes a los tres enfoques de aprendizaje considerados en la investigación y la consiguiente coherencia interna de cada documento.

\section{Tabla 9}

Nivel de coherencia interna de los diseños curriculares con enfoque de aprendizaje no explícito.

\begin{tabular}{cccccccccc}
\hline & \multicolumn{3}{c}{ Ch } & \multicolumn{2}{c}{ Co } & \multicolumn{2}{c}{ Ec } & \multicolumn{2}{c}{ Ve } \\
\cline { 2 - 9 } $\begin{array}{c}\text { Indicadores por enfoque } \\
\text { Currículo }\end{array}$ & & $\mathrm{F}$ & $\mathrm{R}$ & $\mathrm{A}$ & $\mathrm{R}$ & $\mathrm{A}$ & $\mathrm{R}$ & $\mathrm{A}$ & $\mathrm{R}$ \\
Conductistas & 7 & $31 \%$ & 1 & $13 \%$ & 3 & $23 \%$ & 2 & $18 \%$ \\
Cognitivistas & 3 & $14 \%$ & 2 & $25 \%$ & 0 & 0 & 4 & $37 \%$ \\
Constructivista & 12 & $55 \%$ & 5 & $63 \%$ & 10 & $77 \%$ & 5 & $45 \%$ \\
Total indicadores & 22 & $100 \%$ & 8 & $100 \%$ & 13 & $100 \%$ & 11 & $100 \%$ \\
Nivel de coherencia & \multicolumn{2}{c}{ Baja } & \multicolumn{2}{c}{ Alta } & \multicolumn{2}{c}{ Alta } & \multicolumn{2}{c}{ Baja } \\
\hline
\end{tabular}

Ch: Chile, Co: Colombia, Ec: Ecuador, Ve: Venezuela.

Los resultados sintetizados en la Tabla 9, por documento, corresponden a: a) la cantidad absoluta y relativa de indicadores conductistas en los que se encontraron evidencias; b) la cantidad absoluta y relativa de indicadores cognitivistas en los que se encontraron evidencias; c) la cantidad absoluta y relativa de indicadores constructivistas en los que se encontraron evidencias; d) la cantidad del total de indicadores en los que se encontraron evidencias; e) el nivel de coherencia del documento.

El número de indicadores con evidencias en cada documento es: 22 en el de Chile; 8 en el de Colombia; 13 en el de Ecuador y 11 en el de Venezuela. De los cuatro diseños curriculares, los de Chile y Venezuela arrojaron un nivel de coherencia bajo: en ambos casos, el enfoque con evidencias del mayor número de indicadores (el constructivista) agrupa una proporción de indicadores que está entre el $41 \%$ y $60 \%$; el restante $40 \%$ a $59 \%$ de criterios con evidencias se distribuye entre los otros dos enfoques. Específicamente, para el caso de Chile el $55 \%$ corresponde al enfoque constructivista, el $31 \%$ al enfoque conductista y el $14 \%$ al enfoque cognitivista. Para Venezuela el $45 \%$ corresponde al enfoque constructivista, el $37 \%$ al enfoque cognitivista y el $18 \%$ al enfoque conductista.

El documento de Colombia arrojó un nivel de coherencia Alto, ya que el 61\% a 80\% pertenece a un Enfoque y el resto de las evidencias a los otros dos enfoques. De manera detallada el $63 \%$ corresponde al Constructivismo, un $25 \%$ al Cognitivismo y en menor porcentaje al Conductismo con un $13 \%$.

En documento de Ecuador se evidencia en mayor medida las evidencias constructivistas con $77 \%$ y el restante $23 \%$ corresponde al enfoque conductista, obteniendo así un Nivel Alto de Coherencia. 


\section{Conclusiones}

La investigación permite determinar que cinco de los diseños curriculares analizados tienen explícito el enfoque de aprendizaje: los de Argentina, Bolivia, Paraguay, Perú y Uruguay, y tal enfoque es indistintamente el constructivista; asimismo, se determina que los otros cuatro documentos curriculares (los de Chile, Colombia, Ecuador y Venezuela) no tienen explícito el enfoque de aprendizaje y presentan evidencias de más de un enfoque.

En los diseños curriculares cuyo enfoque de aprendizaje no está explícito se observa que la mayor proporción de evidencias corresponden al enfoque constructivista; en el documento de Chile, 12 de 22 indicadores corresponden a dicho enfoque; en el de Colombia, 5 de 8; en el de Ecuador, 10 de 13 y en el de Venezuela, 5 de 11. Ello puede significar que este enfoque, si bien no es el único considerado en el currículo de educación inicial de los países analizados, está teniendo una presencia mayor que los otros dos enfoques considerados en la investigación.

Al observar los niveles de coherencia de los documentos con enfoque explícito se evidencia que aquellos son, en general, mayores que los de los documentos con enfoque no explícito. Todos los diseños curriculares con el enfoque de aprendizaje explícito presentan un nivel de coherencia mayor al nivel bajo, que es el segundo de la escala empleada de cinco niveles; contrariamente, prevalece una alta coherencia en dichos documentos: tres (el 60\%: los de Argentina, Bolivia y Perú) son altamente coherentes con el enfoque explícitamente presentado; y dos (el 40\%: los de Paraguay y Uruguay) muestran ser medianamente coherentes con el mismo. De manera diferente, en los documentos con enfoque no explícito, dos (el 50\%: los de Colombia y Ecuador) muestran una alta coherencia y dos (el otro 50\%: los Chile y Venezuela), presentan una baja coherencia. Puede inferirse que el tener explícito el enfoque de aprendizaje reporta una ventaja en términos de logro de coherencia curricular: sirve a los redactores de los documentos como referencia para desarrollar todos los componentes del currículo de manera afín a su enfoque manifiesto.

Con base en lo anterior, puede sostenerse que, en la elaboración de diseños curriculares resulta conveniente presentar de manera explícita el enfoque de aprendizaje en el que se sustenta; y en ello puede resultar de particular provecho el que no solamente se mencione el enfoque, sino que se expongan los principios constitutivos del mismo. Tales principios servirían de orientación a los diseñadores para la precisión coherente de los planteamientos orientadores y metodológicos del currículo. 
Arancibia, E. Blanco, R. y Avilés, M. (2004). Aprendiendo de las experiencias. Reforma Curricular de la Educación Parvularia. [Versión digital] Santiago: Trineo. Recuperado de http://unesdoc. unesco.org/images/0013/001367/136727s.pdf

Badilla, E. (2009). Diseño Curricular: de la integración a la complejidad. Actualidades educativas en investigación, 9(2), 1-13. Recuperado de http://bit.ly/2zgnXLu

Blanco, M. (2005). La Educación de calidad para todos empieza en la primera infancia. Consideraciones para el desarrollo de políticas educativas. Enfoques Educacionales, 7(1), 1133. Recuperado de https://dialnet.unirioja.es/servlet/articulo?codigo $=1458739$

Cáceres, P. (2003). Análisis cualitativo de contenido: una alternativa metodológica alcanzable. Psicoperspectivas, 2, 53-82. Recuperado de http://www.psicoperspectivas.cl/index.php/ psicoperspectivas/article/viewFile/3/3

Cadena, C. G., \& Saucedo, L. C. (2016). A hundred years away (1913-2013) from the beginning fo watsonian behaviorisim. Revista Interamericana De Psicología, 50(2), 265-274. https://doi. org/10.30849/rip/ijp.v50i2.102

Carretero, M. (2009). Constructivismo y Educación. Buenos Aires: Editorial Paidós.

Casanova, M. (2012). El Diseño Curricular como factor de calidad educativa. Revista lberoamericana sobre calidad, eficacia y cambio educación, 10(4), 6-20. Recuperado de http://www.rinace. net/reice/numeros/arts/volionum4/art1.htm

Castañeda, M., Castro, F. \& Mena, C. (2012). Instrumentos para evaluar el currículum formal en carreras pedagógicas. Panorama, 6 (10), 71-85 Recuperado de https://dialnet.unirioja.es/ servlet/articulo?codigo $=4780122$

Cormack, M. (2004). Estrategias de Aprendizaje y de Enseñanza en la Educación del menor de 6 años. Acción pedagógica, 13(2). Recuperado de https://dialnet.unirioja.es/servlet/ articulo?codigo $=2970397$

Edmonds, W. \& Kennedy, T. (2013). An Applied Reference Guide to Research Designs. Quantitative, Qualitative, and Mixed Methods. Los Angeles: SAGE.

Ertmer, P. \& Newby, T. (2013). Behaviorism, Cognitivism, Constructivism: Comparing Critical Features from an instructional design. Perspective Performance Improvement Quarterly, 26(2), 43-71. https://doi.org/10.1002/piq.21143

Escobar, J. \& Cuervo, A. (2008). Validez de contenido y juicio de expertos: una aproximación a su utilización. Avances en Psicometría, 8, 27-36. Recuperado de

http://www.humanas.unal.edu.co/psicometria/revista/volumenes/volumen-6/

Falabella, A., Cortázar, A., Godoy, F., González, M. P., \& Romo, F. (2018). Sistemas de aseguramiento de la calidad en Educación Inicial: Lecciones desde la experiencia internacional. Gestión y Política Pública, 27(2), 309-340. Recuperado de http://www.gestionypoliticapublica.cide. edu/ojscide/index.php/gypp/article/view/469

Freire, J., Páez, M., Núñez, M., Narváez, M. \& Infante, R. (2018). El diseño curricular, una herramienta para el logro educativo. Revista de Comunicación de la SEECl, 22(45), 75-86. https://doi.org/10.15198/seeci.2018.45.75-86

Guerrero, T. \& Flores H. (2009). Teorías del aprendizaje y la instrucción en el diseño de materiales didácticos informáticos. Educere, 13(45), 317-329. Recuperado de http://www.saber.ula.ve/ handle/123456789/29266

Gutiérrez Ruiz, K. (2015). Perfil agentivo de estudiantes con bajo rendimiento académico: estrategias cognitivas y de control del aprendizaje, autoeficacia académica y motivación. Informes Psicológicos, 15(1), 63-81. http://dx.doi.org/10.18566/infpsicv15n1ao4

Hernández, R., Fernández, C. y Baptista, P. (2010). Metodología de la Investigación. 5ta. Edición. Ciudad de México: Mc Graw Hill. 
Hernández, G. (2002). Paradigmas en psicología de la educación. Ciudad de México: Paidós.

Inciarte, A. y Canquiz, L. (2001). Análisis de la Consistencia Interna del Currículo. Informe de Investigaciones, 15(1 y 2), 79-90. Recuperado de http://biblo.una.edu.ve/ojs/index.php/IIE/ article/viewFile/128/119

Jiménez, L. (2008). Enfoque curricular centrado en la persona. Revista Educación, 32 (1), 63-76. Recuperado de https://revistas.ucr.ac.cr/index.php/educacion/article/view/524

Koumakhov, R., \& Daoud, A. (2017). Routine and reflexivity: Simonian cognitivism vs practice approach. Industrial Q Corporate Change, 26(4), 727-743. https://doi.org/10.1093/icc/dtwo 48

Luzón Trujillo, A. \& Montes Moreno, S. (2018). Perspectiva histórica de la formación inicial del profesorado de Educación Infantil y Primaria en España. Una tarea inacabada. Historia Caribe, 13(33), 121-152. https://doi.org/10.15648/hc.33.2018.6

Manterola, C. (2012). Currículo. Qué y cómo enseñar. Caracas: Editorial Laboratorio Educativo.

Martínez Carpio, H. E. (2013). El enfoque por competencias desde la perspectiva del desarrollo humano. Aspectos básicos y diseño curricular. Avances En Psicología, 21(1), 9-22. Recuperado de http://revistas.unife.edu.pe/index.php/avancesenpsicologia/article/view/302

Marugán, M., Martín, L. J., Catalina, J., \& Román, J. M. (2013). Estrategias cognitivas de elaboración y naturaleza de los contenidos en estudiantes universitarios. Psicologia Educativa, 19(1), 132o. https://doi.org/10.5093/ed2013a3

Mata, Y. (2015). Procedimiento para la evaluación de la pertinencia interna y externa de un currículo en Venezuela. Educ@ción en Contexto, 1(1), 57-77. Recuperado de https://dialnet. unirioja.es/servlet/articulo?codigo $=6296655$

Ministerio de Educación de Bolivia. (2012). Diseño Curricular para el Nivel de Educación Inicial. La Paz: Autor. Bolivia. Recuperado de http://magisteriodebolivia.blogspot.com.uy/p/blogpage.html

Ministerio de Educación de Colombia. (2017). Bases Curriculares para la Educación Inicial y Preescolar. Bogotá: Autor. Recuperado de https://www.mineducacion.gov.co/1759/ articles-341880_recurso_1.pdf

Ministerio de Educación de Chile. (2018). Bases Curriculares de la Educación Parvularia. Santiago de Chile: Autor. Recuperado de https://parvularia.mineduc.cl/equipo-de-aula/curriculum/

Ministerio de Educación de Ecuador. (2014). Currículo de Educación Inicial. Quito: Autor. Recuperado de: https://educacion.gob.ec/oferta-educativas/

Ministerio de Educación y Cultura de Paraguay. (2005). Marco Curricular de la Educación Inicial. Asunción: Autor. Recuperado de https://www.mec.gov.py/cms/?ref=294976-publicacionesde-educacion-inicial

Ministerio de Educación de Perú. (2005). Currículo Nacional de la Educación Básica. Lima: Autor. Recuperado de http://www.minedu.gob.pe/curriculo/

Ministerio de Educación y Cultura de Uruguay. (2014). Marco Curricular para la atención y educación de niñas y niños uruguayos desde el nacimiento a los seis años. Montevideo: Autor. Recuperado de https://mcrn.anep.edu.uy/node/31

Ministerio del Poder Popular para la Educación de Venezuela. (2013). Guía pedagógica-didáctica Educación Inicial Etapa Preescolar. Caracas: Autor. Recuperado de https://www.guao.org/ biblioteca/educacion_inicial_guia_pedagogica_didactica_etapa_preescolar

Poggioli, L. (1997). Estrategias cognoscitivas: una perspectiva teórica. Serie Enseñando a aprender. Caracas: Fundación Polar.

Salas Perea, R. S. (2016). ¿El rediseño curricular sin evaluación curricular es científico? Revista Cubana de Educación Médica Superior, 30(2), 170-180. Recuperado de https://www. medigraphic.com/cgi-bin/new/contenido.cgi?IDPUBLICACION=6713 
Sánchez, G. Á. (2015). Metodología para el diseño curricular en los Programas Nacionales de Formación (PNF). Espacio Abierto. Cuaderno Venezolano de Sociología, 24(4), 129-150. Recuperado de http://www.redalyc.org/articulo.oa?id=12243813007

Secretaría de Educación de la Ciudad Autónoma de Buenos Aires, Argentina (2000). Diseño Curricular para la Educación Inicial. Marco General. Buenos Aires: Autor. Recuperado de: http://www.buenosaires.gob.ar/educacion/docentes/curriculum/nivel-inicial/disenoscurriculares

Serrano, J. M. y Pons, R. M. (2011). El constructivismo hoy: enfoques constructivistas en educación. Revista Electrónica de Investigación Educativa, 13(1). Recuperado de http://redie.uabc.mx/ volizno1/contenido-serranopons.html

Schunk, D. (2012). Teorías del aprendizaje. Una perspectiva educativa. 6ta Edición. Ciudad de México: Pearson Educación.

Terigi, F. (2002). Análisis comparativo de los currículos Iberoamericanos: procesos, condiciones y tensiones que debemos considerar. Organización de Estados Iberoamericanos para la Educación, la Ciencia y la Cultura. Documento presentado en el IV Encuentro Internacional de Educación Inicial y Preescolar "El Currículo y los retos del nuevo milenio", La Habana, Cuba. Recuperado de http://www.oei.es/inicial/articulos/

Unicef (1989). Convención sobre los derechos del niño. Madrid: Autor. Recuperado de https://www. un.org/es/events/childrenday/pdf/derechos.pdf

Unicef (1990) First Call for Children: World Declaration and Plan of Action from the World Summit for Children. New York: Autor. Recuperado de

https://www.unicef.org/spanish/about/history/index_worldsummit.html

Valdez,F. (2012). Teorías educativas y su relación con las tecnologías de la información y de la comunicación (TIC). En XVII Congreso de Administración, Contaduría e Informática. Congreso llevado a cabo en Ciudad Universitaria, México, D.F. Recuperado de http:// congreso.investiga.fca.unam.mx/docs/xvii/docs/L13.pdf

WCEFA (1990). Declaración Mundial sobre Educación para Todos y Marco de Acción para Satisfacer las Necesidades Básicas de Aprendizaje. Jomtien: Autor. Rescatado de https://unesdoc. unesco.org/ark:/48223/pfoooo127583_spa 